\title{
Hydrogel-By-Design: Smart Delivery System for Cancer Immunotherapy
}

\author{
Rongwei Cui ${ }^{1,2}$, Qiang $W_{u^{1}}$, Jing Wang ${ }^{3}$, Xiaoming Zheng ${ }^{1}$, Rongying $\mathrm{Ou}^{4}$, Yunsheng $\mathrm{Xu}^{5}$, \\ Shuxin $Q u^{2 *}$ and Danyang $\mathrm{Li}^{1 *}$ \\ ${ }^{1}$ Research Center, The Seventh Affiliated Hospital, Sun Yat-sen University, Shenzhen, China, ${ }^{2}$ Key Lab of Advanced Technologies \\ of Materials, Ministry of Education, School of Material Science and Engineering, Southwest Jiaotong University, Chengdu, China, \\ ${ }^{3}$ School of Life Sciences, Northwestern Polytechnical University, Xi'an, China, ${ }^{4}$ Department of Gynaecology and Obstetrics, The \\ First Affiliated Hospital of Wenzhou Medical University, Wenzhou, China, ${ }^{5}$ Department of Dermatovenereology, The Seventh \\ Affiliated Hospital, Sun Yat-sen University, Shenzhen, China
}

\section{OPEN ACCESS}

Edited by:

Junqing Wang,

School of Pharmaceutical Sciences (Shenzhen) Sun Yat-sen University,

China

Reviewed by: Rongrong Jln, Sichuan University, China Changqiang $\mathrm{Wu}$,

North Sichuan Medical College, China

*Correspondence:

Shuxin Qu qushuxin@swjtu.edu.cn

Danyang LI

lidy55@mail.sysu.edu.cn

Specialty section:

This article was submitted to Biomaterials,

a section of the journal Frontiers in Bioengineering and Biotechnology

Received: 10 June 2021 Accepted: 09 July 2021

Published: 21 July 2021

Citation:

Cui R, Wu Q, Wang J, Zheng X, Ou R, Xu Y, Qu S and Li D (2021) HydrogelBy-Design: Smart Delivery System for

Cancer Immunotherapy.

Front. Bioeng. Biotechnol. 9:723490.

doi: $10.3389 /$ fbioe.2021.723490
Immunotherapy has emerged as a promising strategy for cancer treatment, in which durable immune responses were generated in patients with malignant tumors. In the past decade, biomaterials have played vital roles as smart drug delivery systems for cancer immunotherapy to achieve both enhanced therapeutic benefits and reduced side effects. Hydrogels as one of the most biocompatible and versatile biomaterials have been widely applied in localized drug delivery systems due to their unique properties, such as loadable, implantable, injectable, degradable and stimulus responsible. Herein, we have briefly summarized the recent advances on hydrogel-by-design delivery systems including the design of hydrogels and their applications for delivering of immunomodulatory molecules (e.g., cytokine, adjuvant, checkpoint inhibitor, antigen), immune cells and environmental regulatory substances in cancer immunotherapy. We have also discussed the challenges and future perspectives of hydrogels in the development of cancer immunotherapy for precision medicine at the end.

Keywords: hydrogels, smart delivery, cancer immunotherapy, immunomodualtors, immune cells, environmental regulatory substance

\section{INTRODUCTION}

In the past decade, cancer immunotherapy has shifted the paradigm for the treatment of cancers that it activates patients' own immune systems to attack and kill cancers. Till now, cancer immunotherapy includes five main classes, immune checkpoint blockade (ICB) therapy, lymphocyte promoting cytokine therapy, chimeric antigen receptor T-cell (CAR-T) therapy, agonistic antibodies and cancer vaccines (Chao et al., 2019; Tang et al., 2021; Yang et al., 2020). The success of those therapies relies on the administration of corresponding biomacromolecules such as checkpoint inhibitors, cytokines, antibodies or T cells and vaccines (Li et al., 2020; Tang et al., 2021). However, high dose or multiple injections involved in conventional drug administration methods may introduce many problems such as safety issues and efficacy (Chao et al., 2019). For instance, the toxicity caused by the delivery agents and the complex tumor microenvironments are still challenges for the development of suitable delivery systems for cancer immunotherapy (Vigata et al., 2020). Therefore, novel delivery approaches could increase the accumulation of immune therapeutics in targeted sites, enabling more effective treatment and reduced side effects (Qiu et al., 2020). Biomaterials such as lipids (Yang et al., 2020), polymers (Munoz et al., 2021), polypeptides and hydrogels (Hoffman, 2012; Munoz et al., 2021) have played vital roles as smart delivery systems for cancer immunotherapy. The unique physicochemical properties of hydrogel have attracted broad attentions of researchers (Hoffman, 2012; Pal et al., 2013). 
Firstly, hydrogels are formed by hydrophilic polymer chains absorbing large amounts of water (Ahmed, 2015; Oliva et al., 2017). The water content could reach $90 \%$, which provides a suitable physiological environment for the cargoes (Akhtar et al., 2016; Xie et al., 2021). Hydrogels with excellent biocompatibility highly mimic the properties of nature tissues because of their high water content (Salah et al., 2020). Furthermore, the softness and rubbery properties of hydrogels minimize inflammatory reaction of surrounding cells. Especially for CAR-T therapy, cell viability could be guaranteed which is the basis for its immune function (Palucka and Banchereau, 2012; Seliktar, 2012; Monette et al., 2016; Zeng et al., 2021). Secondly, the availability of functional groups and the adjustable of the formed network structure of hydrogels allow carrying cargoes with various types and via different magnitude of interaction forces (Qiu et al., 2020). For example, Leach et al. developed an injectable multi-domain peptide-based hydrogel that can carry cyclic dinucleotides and STING (stimulator of interferon genes) agonists through electrostatic interactions (Leach et al., 2018). Additionally, the injectable properties of hydrogels through quick sol-gel phase transition or in situ chemical polymerization render them implantable in the body with minimal surgical wounds and the in situ entrapment of therapeutic molecules or cells by simple syringe injection at target sites (Overstreet et al., 2012; Leach et al., 2019; Vigata et al., 2020). Yu et al. summarized the status and development trend of injectable hydrogel-based cancer immunotherapy and claimed that injectable hydrogels have unprecedented potential to realize the inhibition of tumor growth at the systemic level and prevent the occurrence of side effects through local administration (Chao et al., 2019). Moreover, hydrogels respond immediately to various external stimuli and undergo morphological changes, which realize sustained and controlled release of therapeutics for cancer immunotherapy (Lin and Metters, 2006; Ma et al., 2021). Novel $\mathrm{pH}$-responsive hydrogels with high swelling property at acidic $\mathrm{pH}$ were shown to release drug at a relative fast rate by adjusting the ratio of chitosan and alginate polymers with poly (vinyl alcohol) (Andrade et al., 2021). In addition, the advantages of ease of synthesis, and low raw material prices also make hydrogels a very promising delivery agent for multiple immunological therapeutics (Ahmed, 2015).

This review concentrates on the state-of-the-art of hydrogels applied in cancer immunotherapy as efficient, biocompatible, controllable local drug delivery platforms. The following sections discussed the design principles of hydrogels for delivery therapeutics for cancer immunotherapy, which include the choice of polymer feedstock, the strength of the interaction force between the polymer network, the polymer modification etc. In addition, specific applications of hydrogels in cancer immunotherapy that classified by the carrying cargos which includes cytokines, adjuvant, checkpoint inhibitors, antigen, cells and environmental regulatory substance were discussed and summarized. At last, the development trend, main challenges and clinical application potential of hydrogel-based delivery systems for cancer immunotherapy were discussed.

\section{CHARACTERISTICS OF HYDROGELS AS DELIVERY SYSTEMS FOR CANCER IMMUNOTHERAPY}

Cancer immunotherapy mainly aims to achieve the following purposes, e.g., enhancing the functions of antigen presenting cells, promoting the production of protective T-cells and overcoming immunosuppression (Zou, 2006; Tang et al., 2021). The corresponding therapeutics commonly used in cancer immunotherapy, such as biologically antigenic proteins or peptides, nucleic acids, natural or synthetic adjuvants, and immune cells require the delivery systems to retain their activities and functions (Zhang et al., 2018; Chao et al., 2019).

Hydrogel is a particularly appealing type of drug delivery system because of its unique characters, providing the delivered immunological therapeutics with a physiological environment that highly mimics the natural conditions (Figure 1; Hori et al., 2008; Hickey et al., 2019). These desirable properties include loadable (Figure 2A), implantable/injectable (Figure 2B), degradable (Figure 2C) and stimulus-responsible (Figure 2D). Hydrogels as drug delivery systems can be divided into two categories according to their component sources: synthetic and natural (Chao et al., 2019). Commonly used synthetic hydrogels are hydroxyethyl methacrylate (pHEMA), poly(vinyl alcohol) (PVA), and poly(ethylene glycol) (PEG). These components often have stimulus response characteristics such as temperature sensitivity, so that the hydrogel can be polymerized or degraded in situ (Rizzo and Kehr, 2021). Natural hydrogels include polysaccharides (such as hyaluronic acid and alginate), proteins (such as gelatine and collagen), and DNA. This type of hydrogel has extremely high biocompatibility. The large number of hydroxyl, carboxyl and amino groups in the composition also makes it capable of loading a variety of drugs (Choudhury et al., 2018; Hauptstein et al., 2020). In this part, we focused on the key characters of hydrogels influencing the delivery of therapeutics for cancer immunotherapy.

\section{Loadable}

First of all, hydrogel has the capability to easily encapsulate hydrophilic drugs due to its high water content. The water content of the hydrogel is related to the type of polymers. Natural polymers such as hyaluronic acid-based hydrogel can achieve 99\% water content (Tu et al., 2019; Dorishetty et al., 2020). Secondly, the crosslinked network of hydrogel could prevent the drugs from leaking out and impede penetration of various proteins that may degrade the loaded biological drugs (Pal et al., 2013). This feature means the hydrogel can be loaded with highly labile macromolecular drugs (for example, recombinant proteins and monoclonal antibodies). According to David J. Mooney, the space between polymer molecules in the network (referred to as the mesh size) is tunable from around 5 to around $100 \mathrm{~nm}$ (Li and Mooney, 2016). Importantly, the network can retain the drugs in the "mesh" before the state of the hydrogel changes. The mesh size of hydrogels can be adjusted by the molecular weight and concentration of the polymer (Figure 2A). In general, the higher the molecular weight and the concentration, the smaller the mesh size can be achieved to 


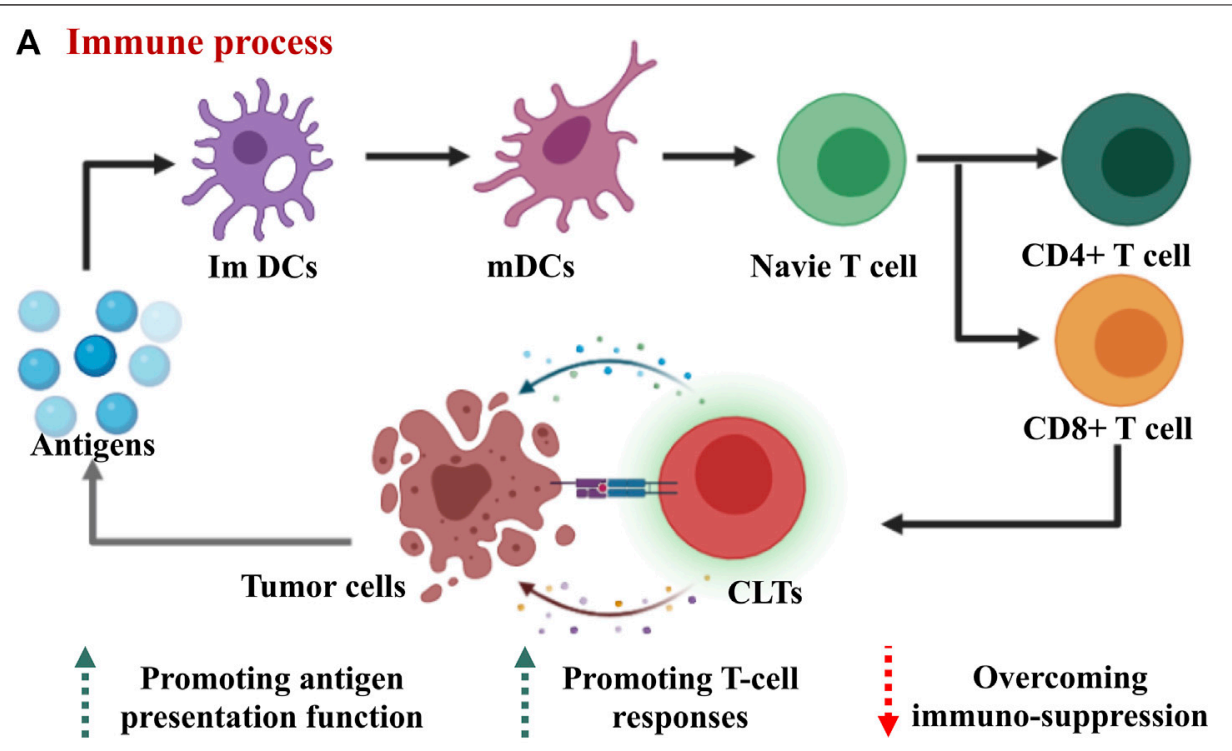

B Hydrogel applied in immunotherapy

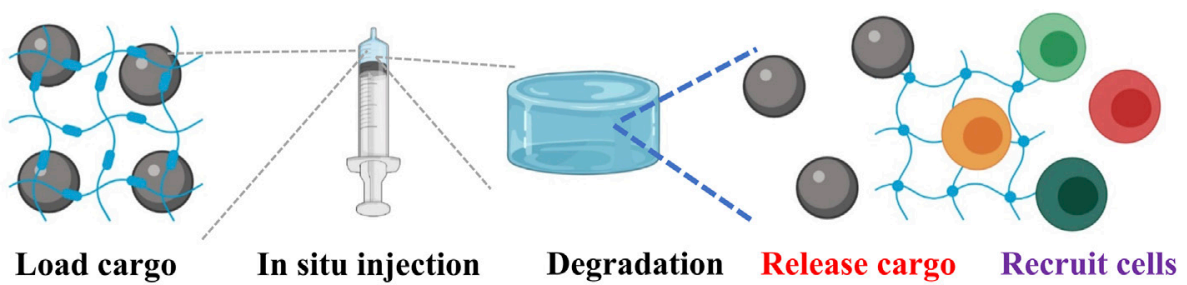

FIGURE 1 | Overview scheme of hydrogels applied in cancer immunotherapy. (A) The schematic diagram of the immune response in the body: antigens are processed by immature dendritic cells (ImDCs), then mature dendritic cells (mDCs) presented antigens to $T$ cells. CD8 ${ }^{+} \mathrm{T}$ cells further differentiate into cytotoxic $T$ lymphocytes (CTLs) to directly kill tumor cells. (B) hydrogels applied in immunotherapy: the hydrogel carrying various cargos is injected into the tumor site and then undergoes degradation, thereby releasing drugs and recruting immune cells to tumor site.

encapsulate the smaller size of drugs. Finally, there are various interactions (Figure 2A) between the drugs and the polymer chains which could be designed via diverse strategies such as covalent conjugation, electrostatic interactions and hydrophobic associations ( $\mathrm{Li}$ and Mooney, 2016). The interactions between hydrogels and drugs are important to maintain the functions and activities of the loaded cargos when designing hydrogels. For instance, Dror et al. claimed the bioactivities of hydrogels could be achieved through immobilizing growth factors and/or small molecules such as RGD peptide (Arginine-Glycine-Aspartic acid) to the backbone polymer. With facilitainge the interactions with cellular and extracellular biomolecules, the hydrogel can also carry immune cells for therapeutic purposes(Chao et al., 2019; Li et al., 2020).

\section{Implantable}

Immunotherapeutics on their own typically require repeated high-dose injections that often result in off target effects with limited efficacy. Implantable scaffolds formed by hydrogel possess powerful synergies with anti-cancer immunotherapies because they allow for discrete localization and controlled release of immunotherapeutic agents, which have been shown various applications to improve outcomes in the treatment of cancers via immunotherapy (Buwalda et al., 2014; Rizzo and Kehr, 2021). Among various strategies, implantable hydrogel can form robust tablet-like scaffolds that are surgically implanted into a tumor resection site, releasing immunoregulatory "cargos" and recruiting immune cells into the porous matrix of the scaffold for further biological programming (Li et al., 2020). In order to prepare hydrogels into implantable scaffold with different mechanical, degradation, and slow-release properties, the cross-linking process needs to be adjusted. Because of the covalent bonds presented between the polymer chains, chemical cross-linking is one of the highly resourceful methods for the formation of hydrogels with excellent mechanical strength (Tie et al., 2020). The hydrogel formed in this method usually degrades slower, so that the release rate of the carried immune drug is relatively slow. Simultaneously, literatures show that hydrogel with a large elastic modulus is beneficial for adhesion and spread of immune cells on its surface, while not being conducive for migration of immune cells into its three-dimensional interior of the hydrogel (Seliktar, 2012; 


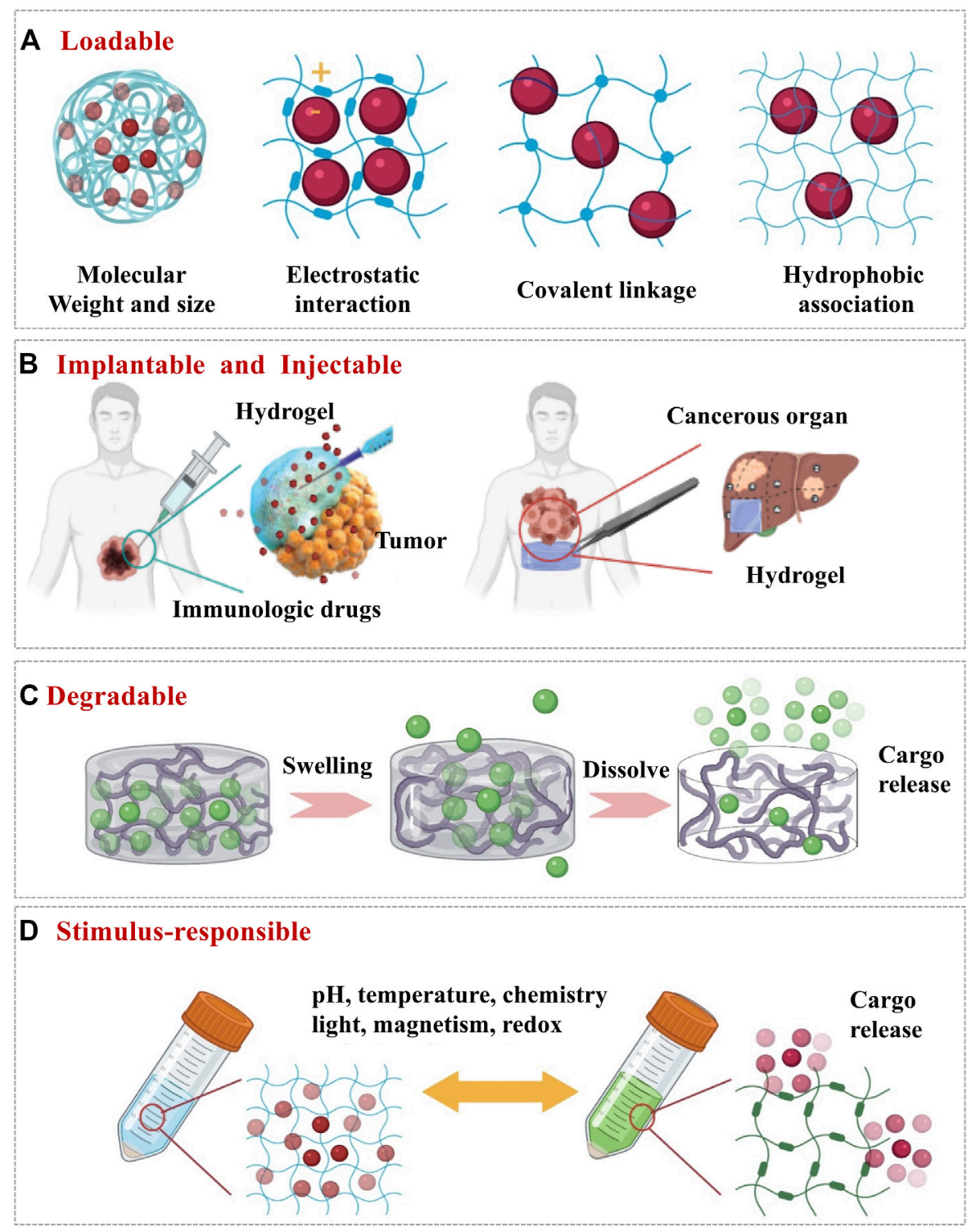

FIGURE 2 | The design of hydrogel by altering its physicochemical properties as delivery systems for cancer immunotherapy. (A) Loadable: ensure that the immune cargo can stay in the hydrogel when it does not need to be released. (B) Implantable and injectable: ensure that the hydrogel can reach the tumor site in a minimally invasive, non-surgical way and stays at the tumor site for drug release. (C) Degradable: hydrogel degrades for releasing the encapsulated drugs. (D) Stimulusresponsible: to achieve controlled release of the drugs from hydrogel upon external stimuli.

Ahearne, 2014; Simona et al., 2015). Since physical cross-linking is relatively mild and avoids the use of cross-linking agents, it is much more friendly to loaded immunotherapy-related biologically active components such as cytokines and immune cells. The interaction forces formed between physical cross-links include hydrogen bonds, hydrophobic interactions and ionic interactions. These weak interaction forces are extremely easy to be destroyed, which makes the drug delivery system easy to degrade and results in relatively rapid drug release (Buwalda et al., 2014). The most advanced application of implantable hydrogels in immunotherapy is enhance adoptive $\mathrm{T}$ cell (ATC) immunotherapy because it is able to support $\mathrm{T}$ cell viability and localize the desired immune response (Oh et al., 2016). Stephan et al. synthesized a calcium cross-linked alginate scaffold containing synthetic collagen mimic peptides, which can promote the adhesion and migration of $\mathrm{T}$ cells and also increase the expansion and dispersion of $\mathrm{T}$ cells to the tumor resection bed (Hori et al., 2008; Rastogi and Kandasubramanian, 2019).

\section{Injectable}

Injectable hydrogels formed in situ by chemical polymerization or sol-gel phase transition have been widely concerned in drug delivery and cancer immunotherapy (Chao et al., 2019). 
Injectable means the materials are flowable before injection but rapidly turn into gels once injected (Figure 2B). Such materials have been widely used because of a few distinct advantages (Rizzo and Kehr, 2021). Firstly, they can be positioned and delivered following the needle, which is a simpler and less traumatic process that avoids unnecessary tissue damage and complications related to inflammation. Secondly, with their viscoelastic properties, injectable materials can move and flow, conforming to any available space before forming into a persisting implant. Koshy et al. reported an injectable gelatin cryogels derived from natural collagen, which were shown to be easily administered without a surgical procedure, promoting infiltration and proliferation of immune cells with controlled release of granulocyte-macrophage colony-stimulating factor (GM-CSF) (Duong et al., 2020). Thirdly, it is easy to encapsulate cells or drugs.

At present, rapid construction of injectable and biocompatible hydrogels with proper mechanical properties remains a challenge. Many methods including thermal gelation, ionic interaction, physical self-assembly and photopolymerization have been employed for preparation of injectable hydrogels in situ (Lei and Tang, 2019; Duong et al., 2020). The "click" reaction, with easy preparation and rapid reaction rate, has emerged as one of the most versatile and innovative technologies (Lei and Tang, 2019). Due to the fast reaction rate and mild reaction conditions, click chemistry is often used to fabricate bio-orthogonal hydrogels, which are highly suitable as carriers for delivering immunotherapy related drugs. In a study by Xu et al., two types of biodegradable and injectable hydrogels using thiol-Michael addition reaction were found to be capable of carrying cells and allowing them function when reaching the designated site (Liu et al., 2020). In addition, Brudno et al. confirmed that utilizing biorthogonal click chemistry to link the functionalized hydrogel with modified anti-cancer pro-drug agents in situ (Ma et al., 2021). In clinical immunotherapy, injectable and implantable can exist simultaneously once the hydrogel is delivered to the tumor site through the needle, the hydrogel can spontaneously form a high-strength scaffold. Kim et al. have developed an injectable cancer vaccine scaffold based on mesoporous silica rods. The system is an ingenious compromise between implantable stents and injectable biomaterials. When injected subcutaneously, it self-assembles into a unique pocket-like reservoir in situ, allowing extended load factor release (Chao et al., 2019).

\section{Degradable}

When used as drug delivery system in cancer immunotherapy, hydrogels mainly release drugs at tumor sites in two ways: hydrogel degradation and hydrogel swelling (Oliva et al., 2017). As the network degrades, the mesh size increases, allowing drugs to diffuse out of the hydrogel (Figure 2C). Hydrogels undergo local or bulk dissolution via a number of mechanisms (such as hydrolysis, proteolysis, disentanglement, or environmental triggers), whilst degradation can occur in the polymer backbone or at the crosslinks, and is typically mediated by hydrolysis or enzyme activity (Tan and Marra, 2010; Vojtova et al., 2019). The hydrogel can be passively degraded or can be actively degraded in response to a certain stimulus, achieving the controlled release of immune "cargos", which will be discussed in more detail in the next section. Furthermore, the degradation process should also not be too short nor too long because the short degradation of hydrogels will cause the explosive release of the drugs and long degradation which will inactivate the immune drugs or cause inflammation in the body. On the other hand, the biodegradation of hydrogels is essential for biomedical applications where controlled in vivo absorption and/or local lysis is required to facilitate cell movement and morphogenesis. For biodegradability, the most common method is to introduce labile bonds in hydrogels either in the cross-links, or the network backbones. Unstable linkages may be cleaved in physiological conditions, either chemically or enzymatically, typically by hydrolysis. Hu et al. reported that a hyaluronic acid hydrogel carries PD-L1antibody (aPD-L1) which is grafted onto the platelet. When the hydrogel is injected into the tumor site, inflammation will initiate platelet activation and release aPD-L1, which achieves a controlled release (Hu et al., 2021).

\section{Stimulus-Responsible}

Those hydrogels with stimulus-responsive properties can be used as a drug delivery system in cancer immunotherapy, in which drug release is triggered upon external stimulis (Bajpai et al., 2008; Andrade et al., 2021) such as $\mathrm{pH}$, temperature, and biomolecule concentration (Figure 2D). The cross-linking process of the hydrogel can respond to external stimuli. One of the most representative example is the novel thermogelling poly(lactic-co-glycolic acid)-poly(ethylene glycol)-poly(lacticco-glycolic acid) (PLGA-PEG-PLGA) triblock copolymer, which exhibits a sol-gel transition with increasing temperature (Li et al., 2021). Jiang et al. incorporated multifunctional dendritic nanoparticles into a injectable thermosensitive hydrogel matrix (PLGA-PEG-PLGA triblock copolymers) to construct a localized drug delivery system for combining chemotherapy and immunotherapy (Vojtova et al., 2019). It is noteworthy that the dendritic nanoparticles-in-hydrogel delivery system gels at physiological temperature, thus allowing for sustained release of drugs. Ultimately, this system showed remarkable efficacy in treating triple negative breast cancer in mice model with $86 \%$ tumor growth inhibition. Additionally, the degradation process of hydrogel can respond to external stimuli. In response to pathological cues, hydrogel undergoes degradation upon external stimuli, followed by the release of the drugs which was precisely controlled by the pathological need. Gajanayake et al. developed a hydrogel that can release the immunosuppressant tacrolimus responsively to deal with proteolytic enzymes overexpressed in inflammation. Within 14 days, drugs in the hydrogel can be completely released, while the drug release rate in phosphate buffered saline for 28 days is less than $10 \%$. Immunosuppressive drug tacrolimus was encapsulated in hydrogelS responsive to proteolytic enzymes which are overexpressed in inflammation. Within 14 days, complete drug release was achieved without burst release, whilst the drug release in phosphate-buffered saline was below $10 \%$ over 28 days (Oliva et al., 2017). The swelling behaviour of hydrogels can be affected by various external physicochemical 


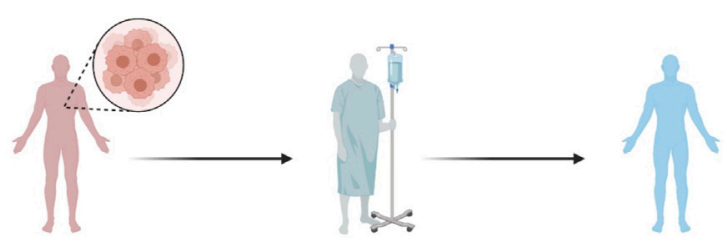

Cancer patient Immunotherapy Healthy patient

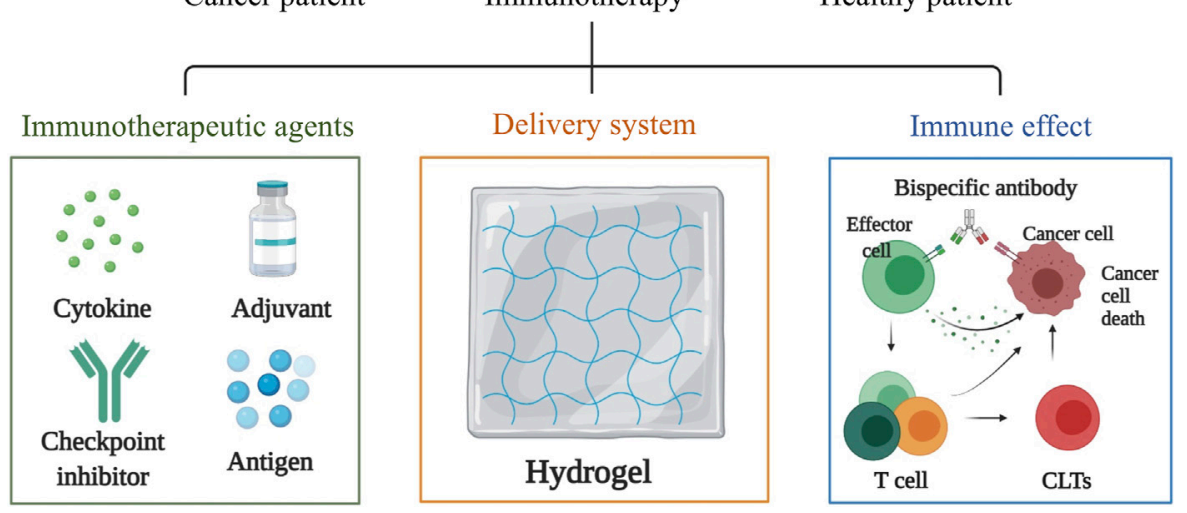

FIGURE 3 | Hydrogels applied in the delivery of immunomodulatory molecules which include cytokine, adjuvant, checkpoint inhibitor and antigen. These immunomodulatory molecules can be loaded to the biocompatible hydrogel in a simple and gentle way to ensure the biological activity of these immunomodulatory factors, enabling them to exert their immune effects.

conditions, which have been widely exploited in controlled drug delivery. $\mathrm{pH}$ responsive swelling is particularly important for cancer delivery systems because it allows targeted drug release in solid tumors where tumour microenvironments are typically more acidic than normal tissues (Andrade et al., 2021). Other stimuli responsive swelling mechanisms have also been exploited for controlled drug delivery. For example, a temperature responsive nanogel was fibricated to deliver the chemotherapy drug cisplatin to breast cancer that the temperature was believed to be slightly higher than the normal ones (Li et al., 2021) In some other cases, the drug carried by the hydrogel responds to stimuli. Natalie et al. incorporated gold nanoparticles decorated with 5fluorouracil (5-FU)-hairpin DNA into dendrimer-dextran hydrogels to silence multidrug resistance protein-1 (MRP1). When the hairpin is opened by hybridizing with a complementary target (MRP1 mRNA), this interaction becomes weaker, allowing simultaneous release of the drug and knockout of the gene encoding the protein responsible for drug resistance (Ding et al., 2019). These smart properties of hydrogels mentioned above make hydrogel attractive and praisable as drug delivery systems for cancer immunotherapy.

\section{APPLICATIONS OF HYDROGELS AS DELIVERY SYSTEMS FOR CANCER IMMUNOTHERAPY}

\section{Delivery of Immunomodulatory Molecules}

A wide variety of immunotherapeutic agents, from small molecule drugs to macromolecular drugs and even cells, can be incorporated into the hydrogel for enhanced cancer immunotherapy. In this section, we discussed the application of hydrogel as delivery system for immunomodulatory molecules such as cytokine, adjuvant, checkpoint inhibitor and antigen (Figure 3).

\section{Cytokine}

In cancer immunotherapy, cytokines can be used to either activate or suppress the immune response against tumors (Chao et al., 2019). For example, Interleukin-12 (IL-12), a heterodimeric cytokine composed of two polypeptides with disulfide linkage, enhances the immune response of Th1, the maturation of cytotoxic $\mathrm{T}$ lymphocytes, the stimulation of natural killer cells, and the production of interferon- $\gamma$ (IFN$\gamma)$. To overcome the main impediment of cytokines' short halflife in vivo, Shimizu et al. encapsulated IL-12 in a cholesterolbearing pullulan (CHP) nanogel. After the mice were subcutaneously injected with murine IL-12-loaded CHP nanogel (CHP/rmIL-12), the concentration of serum rmIL-12 stays relatively high for 12-24 h (Shimizu et al., 2008). GM-CSF, also known as colony stimulating factor 2 (CSF2), is a monomeric glycoprotein that secretes by macrophages, $\mathrm{T}$ cells, mast cells, natural killer cells, endothelial cells, and fibroblasts. Doo Sung Lee et al. have designed an injectable smart hydrogels composed of levodopa and poly( $\varepsilon$-caprolactone-co-lactide)ester-functionalized hyaluronic acid (HA-PCLA) that loaded with OVA expressing plasmid (pOVA) and GM-CSF to boost the maturation of dendritic cells (DCs). By the controlled release of pOVA and GM-CSF through a single subcutaneous injection, the hydrogel system effectively eliminated the B16/OVA melanoma tumors (Leach et al., 2018). 


\section{Adjuvant}

Chemotherapy and radiotherapy can induce immunogenic cell death (ICD), such antitumor immune response would be further amplified if there are concurrent immune adjuvants inside the tumor. Adjuvants are nonspecific immunostimulants, which enhance the body's immune response when combined with antigens. For example, Hou et al. used genetically engineered polypeptide hydrogel PC10ARGD as a sustained-release system that delivered Bestatin which is an immune adjuvant for mammary carcinoma treatment. The hydrogel serves as a drug delivery depot for sustained drug release of Bestatin, which further inhibits tumour metastases and microvessel formation but amplified T lymphocytes function (Hou et al., 2020). Liu et al. developed a smart hydrogel based on alginate, which hybridized with immunoadjuvant $\mathrm{CpG}$ oligonucleotide and conjugated with an adenosine triphosphate (ATP)-specific aptamer. Once intratumoral injection, this hydrogel gelled in situ and released the CpG oligonucleotide, achieving remarkable synergistic tumor elimination as well as generating long-lasting immune memory to prevent recurrences (Wu et al., 2021).

\section{Checkpoint Inhibitor}

Immunological checkpoints are a class of immunosuppressive molecules that can regulate the intensity and duration of immune responses, thereby avoiding damage of normal tissues. During the occurrence and development of tumors, immune checkpoints become one of the main regulators for immune tolerance ( $\mathrm{Yu}$ et al., 2018). Immune checkpoint blockade therapy is an approach that regulates the activity of $\mathrm{T}$ cells through a series of channels such as cosuppression or costimulation signals to kill tumor cells. The most commonly used therapeutic drugs are programmed death receptors 1 /programmed death receptor-ligand 1 (PD-1/ PD-L1) and cytotoxic $\mathrm{T}$ lymphocyte-associated antigen-4 (CTLA-4) inhibitors. Activated $\mathrm{T}$ cells express PD-1 to recognize and remove abnormal or cancerous cells. However, tumor cells inactivate the $\mathrm{T}$ cells that recognize tumor antigens by expressing PD-1 ligands that bind to PD-1, thereby evading immune system attacks. Therefore, tumor cell death can be induced by blocking inhibitors of PD-1 or its ligand. CTLA-4 is another immune checkpoint that reduces T-cell activation and promotes tumor progression by binding to its ligands. Inhibitors against CTLA-4 and its ligands block their interactions to increase T-cell activity and thereby clear tumors (Kazemi et al., 2021; Tang et al., 2021). Immune checkpoint inhibitors can cause side effects to many normal organs. Therefore, a large number of studies have used hydrogel as delivery system to locally release immune checkpoint inhibitors at the tumor site to solve the above problems. Chen et al. developed an anti-inflammatory nanofiber hydrogel self-assembled by steroid drugs for local delivery of ( $\alpha \mathrm{PD}-\mathrm{L} 1)$. Effective therapeutic effects were observed with inhibition both local tumors and abscopal tumors once injected with aPDL1-loaded hydrogel.

\section{Antigen}

Low immunogenicity and tumor heterogeneity greatly limit the therapeutic effect of tumor vaccines. In view of this, Zhu et al. prepared a new type of injectable adhesive hydrogel based on thermosensitive nanogels containing catechol groups and loaded with $\mathrm{MnO}_{2}$ nanoparticles. After intratumoral injection, the concentrated nanogel dispersion transformed into an adhesive hydrogel in situ. The photothermal effect of the loaded $\mathrm{MnO}_{2}$ nanoparticles induces immunogenic cell death and releases a large amount of autologous tumor-derived protein antigens under near-infrared radiation. These antigens captured by the hydrogel are ideal immunostimulatory substances to avoid the consequences of tumor heterogeneity. They also recruit more dendritic cells to stimulate a strong and durable anti-tumor immune response mediated by $\mathrm{CD}^{+} \mathrm{T}$ cells (Song et al., 2018). Wang et al. used polypeptide hydrogel loaded with tumor cell lysates (TCL) as antigens, Toll-like receptors 3 (TLR3) agonist, polyinosinic:polycytidylic acid [poly(I:C)] to construct the vaccines, which robustly recruit, activate and mature DCs in vitro and in vivo by sustained release of TCL and poly (I:C). The antigen persistence at the injection site and antigen drainage to lymph nodes were significantly improved by hydrogel (Song et al., 2018).

\section{Delivery of Immune Cells}

Localized delivery using hydrogels in immunotherapy provides adjustable microenvironments for immune cells as it enables the recruitment, expansion, and activation of immune cells in vitro and in vivo (Figure 4; Weiden et al., 2018; Yang et al., 2018) It is reported that a thermosensitive chitosan hydrogel for the delivery of tumor-infiltrating lymphocytes and activated $\mathrm{CD} 8^{+} \mathrm{T}$ cells. To provide a suitable physical condition for the proliferation of immune cells, the gelation process and mechanical strength of the hydrogel could be precisely tuned by varying the concentration of sodium bicarbonate. The in vitro T-cell expansion experiment revealed that hydrogel platform with a larger pore size and higher stiffness promoted the viability and proliferation of the encapsulated T cells (Hickey et al., 2019). DCs can either be activated in hydrogels in vitro before implantation, or by immobilizing stimuli within the gels in vivo (Yang et al., 2018). Verbeke et al. designed an injectable alginate hydrogel system that allows DCs to be enriched locally in vivo without activation or maturation. Similarly, alginate hydrogels was designed to carry and releas antigen-loaded DCs when injected subcutaneously in mice (Hori et al., 2008). Besides DCs, macrophages also playws a major role as antigen-presenting cells in tumor vaccination. Muraoka et al. developed a cholesteryl pullulan-based hydrogel to deliver peptide-based antigens to macrophages (Chao et al., 2019). The antigens were successfully delivered to $\mathrm{CD}^{+}$cytotoxic $\mathrm{T}$ cells by this immunologically inert hydrogel.

Passive immunotherapy uses components of immune system to target specific cancers without necessarily involving an immune response. Since the first clinical trial 28 years ago, tumor-infiltrating lymphocytes (TILs) have been widely applied for the treatment of metastatic melanoma. Meanwhile remarkable research efforts have been focusd on adopative cell therapy (ACT), which heavily relies on the administration of allogeneic T cells (Monette et al., 2016). Furthermore, genetically modified tumor-specific $\mathrm{T}$ cells have been developed to overcome the limitation of TIL expansion and the augment ACT-mediated 


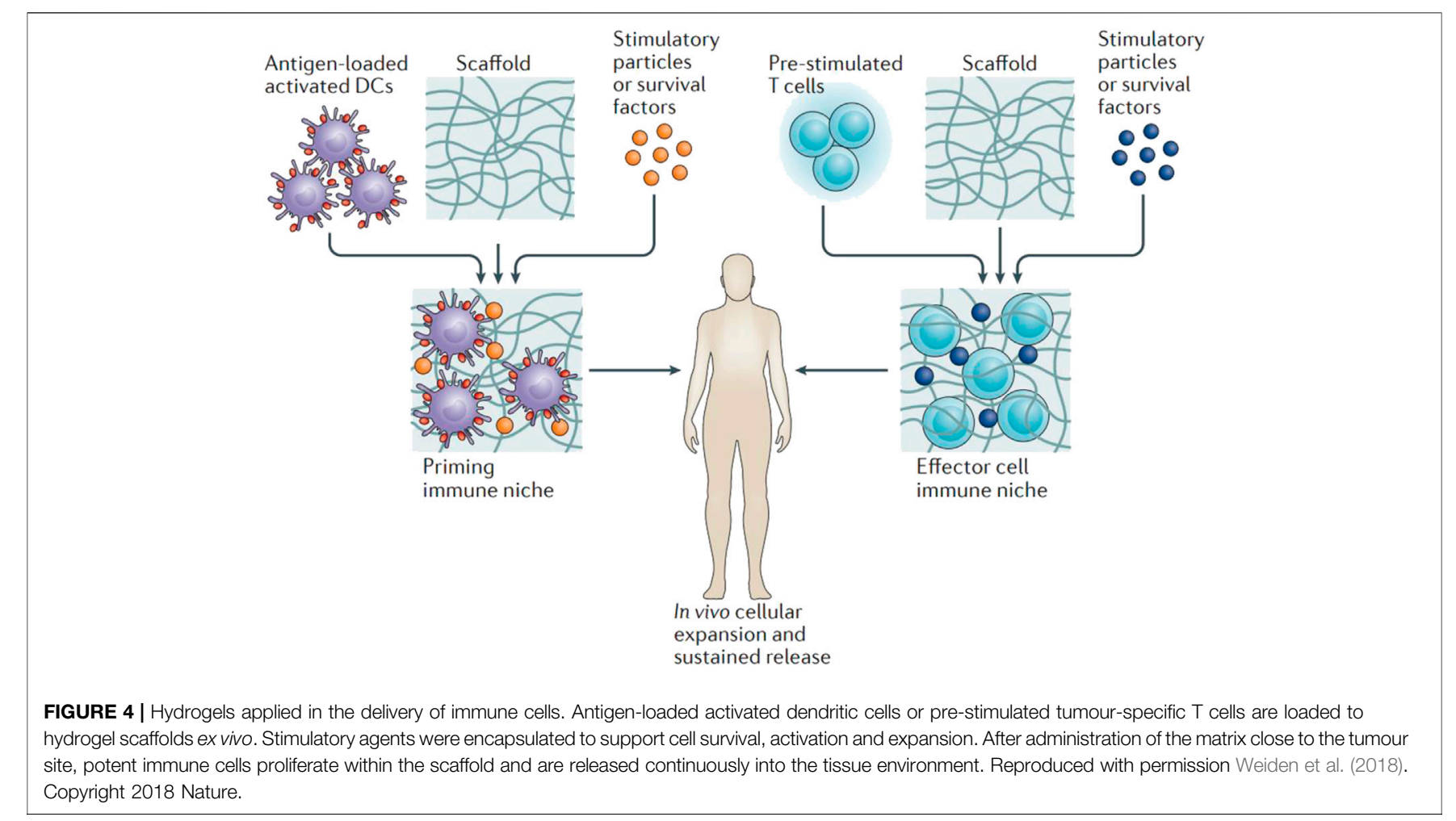

immunotherapy. Compared with systemic delivery, which is likely to result in $\mathrm{T}$ cells infiltrating to noncancerous tissues, localized administration using hydrogels into tumor sites improves delivery efficiency and sustained release of $\mathrm{T}$ cells. The feasibility of a thermosensitive, amine-reactive oligo(ethylene glycol) methacrylate-based hydrogel was investigated as a $\mathrm{T}$ cell carrier. Additionally, an injectable thermogel based on chitosan was developed with the purpose of encapsulating, expanding, and delivering cytotoxic $\mathrm{T}$ cells (Jiang et al., 2018).

\section{Delivery of Environmental Regulatory Substances}

Practically, in addition to carrying the above-mentioned cytokines, immune adjuvants, checkpoint inhibitors, and immune cells that directly stimulate immunity, the hydrogel can also carry environmental regulation substances to regulate the tumor microenvironment. For example, acidic extracellular $\mathrm{pH}$ promotes immune evasion and tumor progression. Therefore, antagonizing tumor acidity can be a powerful approach in cancer immunotherapy. Hyung-seung et al. used Pluronic F-127 as a $\mathrm{NaHCO}_{3}$ releasing carrier to focally alleviate extracellular tumor acidity (Figure 5A). In a mouse tumor model, intratumoral treatment with $\mathrm{pH}$ modulating injectable gel (pHe-MIG) generates immune-favorable TME, as evidenced by the decrease of immune-suppressive cells and increase of tumor infiltrating $\mathrm{CD}^{+} \mathrm{T}$ cells (Jin et al., 2019). It has also been suggested that implantable hydrogel can be engineered to investigate specific aspects of the tumor microenvironment, either singularly or in combination. It is known that lactate, a metabolite byproduct of anaerobic glycolysis, is known to reprogram immune cells, resulting in increased tumor survival. Riley et al. (Figure 5B) designed and synthesized a peptide hydrogel loaded with GM-CSF and poly-(lactic-co-glycolic acid)/(lactic acid) microparticles which can generate the localized lactate concentrations $(\sim 2-22 \mathrm{mM})$ and cellular makeup of the tumor microenvironment, following subcutaneous implantation in mice and causing significant immunological effects (Allen et al., 2020). Jiang et al. incorporated multifunctional dendritic nanoparticles into a injectable hydrogel matrix which is composed with thermosensitive triblock copolymers to construct a localized drug delivery system for combining gas-therapy and immunotherapy (Figure 5C). The dendritic scaffolds were used to deliver arginine-rich molecules (L-Arg) to M1 macrophages, which produce the cytotoxic substance nitric oxide (NO), and subsequently induce tumor cell destruction (Jiang et al., 2018).

\section{CONCLUSION AND PERSPECTIVES}

Immunotherapy is a type of approach that utilizes the body's own immune system to combat cancer, demonstrating to be effective or completely alleviating some solid or hematological malignant tumors. Hydrogels exhibited excellent promise for cancer immunotherapy since they are loadable, implantable, injectable, biodegradable and stimuli-responsive ( $\mathrm{Bu}$ et al., 2019). These properties guarantee hydrogels higher 

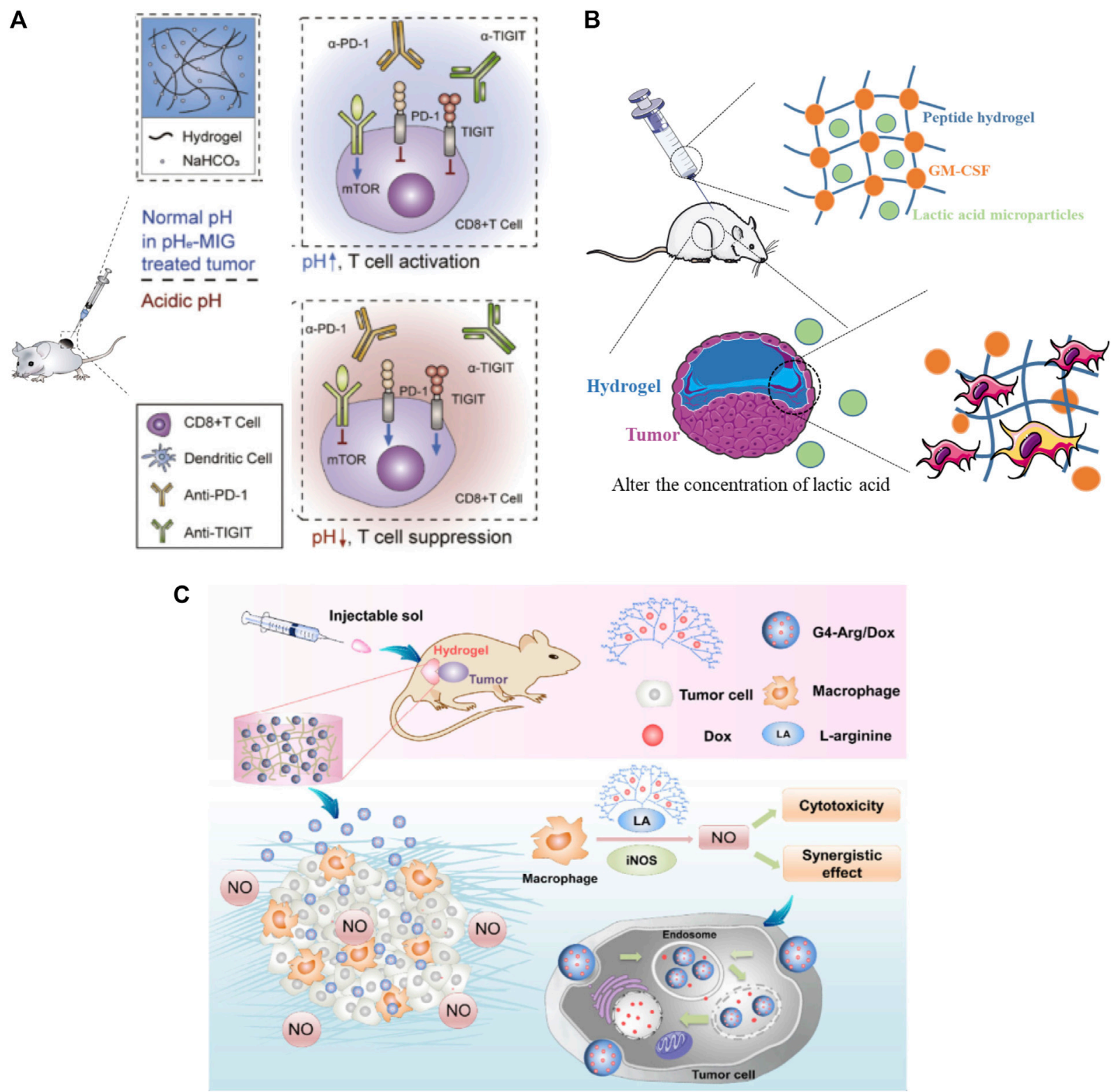

FIGURE 5 | Hydrogels applied in the delivery of environmental regulatory substance. (A) Pluronic $\mathrm{F}-127$ was used as a $\mathrm{NaHCO}_{3}$ releasing carrier to focally alleviate extracellular tumor acidity. Reproduced with permission Jin et al. (2019). Copyright 2019 Elsevier Ltd. (B) A lactic acid-producing hydrogel system was design to change the concentration of lactic acid at the tumor site to interrogate immune cell modulation in cancer-like environments. Reproduced with permission Allen et al. (2020). Copyright. 2020. The Royal Society of Chemistry. (C) A dendritic scaffolds were designed to enrich arginine molecules and provide the inducible nitric oxide synthase (iNOS) substrate, L-Arg, to M1 macrophages, which can produce the cytotoxic substance nitric oxide (NO) and subsequently induce tumor cell destruction through immunotherapy. Reproduced with permission Jiang et al. (2018). Copyright 2018 springer.

immunological drug loading efficiency and drug stability, more controllable drug release profiles, and easier to combine immunotherapy with other treatment methods (Yang et al., 2020). In this review, we focus on how the characteristics of hydrogels have been applied to deliver cytokines, adjuvants, checkpoint inhibitors, antigens, cells, and environmental regulatory substance in cancer immunotheraoy. These reported hydrogel systems are able to prolong the residence time of immunotherapy factors at tumor sites, as well as protect bioactive molecules from denaturation or degradation, thus improving the efficacy of immunotherapy.

In spite of the successful exploration of hydrogel drug delivery systems for cancer immunotherapy, several challenges still need to be considered for the future development and potential applications. Firstly, the controlled release of drug from hydrogels still remains as an issue since immunological drug are released by diffusion and hydrogel degradation, both of which are uncontrollable processes. Those hydrogels are responsive to 
stimulation can release immunological drugs, but the research in this area is only limited to in vitro experiments or rely heavily on the intrinsic tumor microenvironment. Once the hydrogel implanted into the body, it will be important to find out if same type of response properties exists in vivo. Secondly, although hydrogel materials have demonstrated good biocompatibility and biodegradability, higher biocompatibility is needed when used to deliver immunological drugs. In clinical application, the safety of the delivery agents is extremely crucial, particularly in cancer immunotherapy, those bioactive molecules or cells discussed above require an environment that is similar to physiological conditions more than other drugs. Thirdly, there are some issues that need to be considered in clinical aspects: 1) multiple imaging-guided injection techniques should be developed for deep-sited tumors administration; 2) The dose of immune drugs is also a vital parameter. For in vitro experiments, a drug dose is usually chosen based on the effectiveness of the drug. In clinical practice, however, a strict dose screening should be performed according to the drug release curve of the hydrogel. High concentration of anticancer agents can also cause severe acute injury to the healthy parenchyma due to the burst release. If the drug release from the hydrogel does not proceed at a uniform rate, it can also lead to increase the risk of drug resistance; 3) It will be feasible to construct the final system on the basis of approved clinical materials as it is also more likely to get clinical approval.

\section{REFERENCES}

Ahearne, M. (2014). Introduction to Cell-Hydrogel Mechanosensing. Interf. Focus. 4 (2), 20130038. doi:10.1098/rsfs.2013.0038

Ahmed, E. M. (2015). Hydrogel: Preparation, Characterization, and Applications: A Review. J. Adv. Res. 6 (2), 105-121. doi:10.1016/j.jare.2013.07.006

Akhtar, M. F., Hanif, M., and Ranjha, N. M. (2016). Methods of Synthesis of Hydrogels ... A Review. Saudi Pharm. J. 24 (5), 554-559. doi:10.1016/j.jsps.2015.03.022

Allen, R., Ivtchenko, E., Thuamsang, B., Sangsuwan, R., and Lewis, J. S. (2020). Polymer-loaded Hydrogels Serve as Depots for Lactate and Mimic "cold" Tumor Microenvironments. Biomater. Sci. 8 (21), 6056-6068. doi:10.1039/d0bm01196g

Andrade, F., Roca-Melendres, M. M., Durán-Lara, E. F., Rafael, D., and Schwartz, S., Jr. (2021). Stimuli-Responsive Hydrogels for Cancer Treatment: The Role of pH, Light, Ionic Strength and Magnetic Field. Cancers 13 (5), 1164. doi:10.3390/ cancers13051164

Bajpai, A. K., Shukla, S. K., Bhanu, S., and Kankane, S. (2008). Responsive Polymers in Controlled Drug Delivery. Prog. Polym. Sci. 33 (11), 1088-1118. doi:10.1016/ j.progpolymsci.2008.07.005

Bu, L.-L., Yan, J., Wang, Z., Ruan, H., Chen, Q., Gunadhi, V., et al. (2019). Advances in Drug Delivery for post-surgical Cancer Treatment. Biomaterials 219, 119182. doi:10.1016/j.biomaterials.2019.04.027

Buwalda, S. J., Boere, K. W. M., Dijkstra, P. J., Feijen, J., Vermonden, T., and Hennink, W. E. (2014). Hydrogels in a Historical Perspective: from Simple Networks to Smart Materials. J. Controlled Release 190, 254-273. doi:10.1016/j.jconrel.2014.03.052

Chao, Y., Chen, Q., and Liu, Z. (2019). Smart Injectable Hydrogels for Cancer Immunotherapy. Adv. Funct. Mater. 30 (2), 1902785. doi:10.1002/adfm.201902785

Choudhury, D., Tun, H. W., Wang, T., and Naing, M. W. (2018). Organ-Derived Decellularized Extracellular Matrix: A Game Changer for Bioink Manufacturing?. Trends Biotechnol. 36 (8), 787-805. doi:10.1016/j.tibtech.2018.03.003

Ding, F., Huang, X., Gao, X., Xie, M., Pan, G., Li, Q., et al. (2019). A Non-cationic Nucleic Acid Nanogel for the Delivery of the CRISPR/Cas9 Gene Editing Tool. Nanoscale 11 (37), 17211-17215. doi:10.1039/c9nr05233j

Dorishetty, P., Dutta, N. K., and Choudhury, N. R. (2020). Bioprintable Tough Hydrogels for Tissue Engineering Applications. Adv. Colloid Interf. Sci. 281, 102163. doi:10.1016/j.cis.2020.102163
In summary, the delivery systems based on hydrogels have exhibited excellent promises for cancer immunotherapy as the unique characteristics of hydrogels ensure locally and sustainably delivering immunotherapeutic agents to target tumor sites with reduced drug dose and side effects. However, challenges still exist, which requires experts from multidisciplinary fields work more closely. We believe this smart delivery system will continue contributing in tomour threatment via immunotherapy as well as other aspects for improving human health.

\section{AUTHOR CONTRIBUTIONS}

Conception of the work: RC, SQ and DL; Drafting the article: RC and DL; Critical revision of the article: RC, QW, JW, XZ, RO, YX, SQ and DL.

\section{FUNDING}

This work is supported by the following programs: National Natural Science Foundation of China (Grant Nos. 52035012, 81771531, 81871129, 82072862, 82072863, 61805158), the Basic Research Foundation Key Project of Sichuan Province (2021JY0046) and Research Start-up Grant of SAHSYSU (ZSQYBRJH0019).

Duong, H. T. T., Thambi, T., Yin, Y., Kim, S. H., Nguyen, T. L., Phan, V. H. G., et al. (2020). Degradation-regulated Architecture of Injectable Smart Hydrogels Enhances Humoral Immune Response and Potentiates Antitumor Activity in Human Lung Carcinoma. Biomaterials 230, 119599. doi:10.1016/j.biomaterials.2019.119599

Hauptstein, J., Böck, T., Bartolf-Kopp, M., Forster, L., Stahlhut, P., Nadernezhad, A., et al. (2020). Hyaluronic Acid-Based Bioink Composition Enabling 3D Bioprinting and Improving Quality of Deposited Cartilaginous Extracellular Matrix. Adv. Healthc. Mater. 9 (15), 2000737. doi:10.1002/adhm.202000737

Hickey, J. W., Dong, Y., Chung, J. W., Salathe, S. F., Pruitt, H. C., Li, X., et al. (2019). Engineering an Artificial T-Cell Stimulating Matrix for Immunotherapy. Adv. Mater. 31 (23), 1807359. doi:10.1002/adma.201807359

Hoffman, A. S. (2012). Hydrogels for Biomedical Applications. Adv. Drug Deliv. Rev. 64, 18-23. doi:10.1016/j.addr.2012.09.010

Hori, Y., Winans, A. M., Huang, C. C., Horrigan, E. M., and Irvine, D. J. (2008). Injectable Dendritic Cell-Carrying Alginate Gels for Immunization and Immunotherapy. Biomaterials 29 (27), 3671-3682. doi:10.1016/ j.biomaterials.2008.05.033

Hou, X.-l., Dai, X., Yang, J., Zhang, B., Zhao, D.-h., Li, C.-q., et al. (2020). Injectable Polypeptide-Engineered Hydrogel Depot for Amplifying the Anti-tumor Immune Effect Induced by Chemo-Photothermal Therapy. J. Mater. Chem. B 8 (37), 8623-8633. doi:10.1039/d0tb01370f

Hu, Q., Li, H., Archibong, E., Chen, Q., Ruan, H., Ahn, S., et al. (2021). Inhibition of post-surgery Tumour Recurrence via a Hydrogel Releasing CAR-T Cells and AntiPDL1-conjugated Platelets. Nat. Biomed. Eng. doi:10.1038/s41551-021-00712-1

Jiang, L., Ding, Y., Xue, X., Zhou, S., Li, C., Zhang, X., et al. (2018). Entrapping Multifunctional Dendritic Nanoparticles into a Hydrogel for Local Therapeutic Delivery and Synergetic Immunochemotherapy. Nano Res. 11 (11), 6062-6073. doi:10.1007/s12274-018-2123-8

Jin, H.-s., Choi, D.-s., Ko, M., Kim, D., Lee, D.-h., Lee, S., et al. (2019). Extracellular $\mathrm{pH}$ Modulating Injectable Gel for Enhancing Immune Checkpoint Inhibitor Therapy. J. Controlled Release 315, 65-75. doi:10.1016/j.jconrel.2019.10.041

Kazemi, M. H., Najafi, A., Karami, J., Ghazizadeh, F., Yousefi, H., Falak, R., et al. (2021). Immune and Metabolic Checkpoints Blockade: Dual Wielding against Tumors. Int. Immunopharmacology 94, 107461. doi:10.1016/ j.intimp.2021.107461 
Leach, D. G., Dharmaraj, N., Piotrowski, S. L., Lopez-Silva, T. L., Lei, Y. L., Sikora, A. G., et al. (2018). STINGel: Controlled Release of a Cyclic Dinucleotide for Enhanced Cancer Immunotherapy. Biomaterials 163, 67-75. doi:10.1016/ j.biomaterials.2018.01.035

Leach, D. G., Young, S., and Hartgerink, J. D. (2019). Advances in Immunotherapy Delivery from Implantable and Injectable Biomaterials. Acta Biomater. 88, 15-31. doi:10.1016/j.actbio.2019.02.016

Lei, K., and Tang, L. (2019). Surgery-free Injectable Macroscale Biomaterials for Local Cancer Immunotherapy. Biomater. Sci. 7 (3), 733-749. doi:10.1039/c8bm01470a

Li, J., Luo, Y., Li, B., Xia, Y., Wang, H., and Fu, C. (2020). Implantable and Injectable Biomaterial Scaffolds for Cancer Immunotherapy. Front. Bioeng. Biotechnol. 8, 612950. doi:10.3389/fbioe.2020.612950

Li, J., and Mooney, D. J. (2016). Designing Hydrogels for Controlled Drug Delivery. Nat. Rev. Mater. 1 (12). doi:10.1038/natrevmats.2016.71

Li, L., He, Y., Zheng, X., Yi, L., Nian, W., and Abadi, P. P. (2021). Progress on Preparation of $\mathrm{pH} /$ Temperature-Sensitive Intelligent Hydrogels and Applications in Target Transport and Controlled Release of Drugs. Int. J. Polym. Sci. 2021, 1-14. doi:10.1155/2021/1340538

Lin, C.-C., and Metters, A. T. (2006). Hydrogels in Controlled Release Formulations: Network Design and Mathematical Modeling. Adv. Drug Deliv. Rev. 58 (12-13), 1379-1408. doi:10.1016/j.addr.2006.09.004

Liu, Y., Liu, M., Zhang, Y., Cao, Y., and Pei, R. (2020). Fabrication of Injectable Hydrogels via Bio-Orthogonal Chemistry for Tissue Engineering. New J. Chem. 44 (27), 11420-11432. doi:10.1039/d0nj02629h

Ma, H., He, C., and Chen, X. (2021). Injectable Hydrogels as Local Depots at Tumor Sites for Antitumor Immunotherapy and Immune-Based Combination Therapy. Macromol. Biosci. 21, 2100039. doi:10.1002/mabi.202100039

Monette, A., Ceccaldi, C., Assaad, E., Lerouge, S., and Lapointe, R. (2016). Chitosan Thermogels for Local Expansion and Delivery of Tumor-specific $\mathrm{T}$ Lymphocytes towards Enhanced Cancer Immunotherapies. Biomaterials 75, 237-249. doi:10.1016/j.biomaterials.2015.10.021

Muñoz, N. M., Williams, M., Dixon, K., Dupuis, C., McWatters, A., Avritscher, R., et al. (2021). Influence of Injection Technique, Drug Formulation and Tumor Microenvironment on Intratumoral Immunotherapy Delivery and Efficacy. J. Immunother. Cancer 9 (2), e001800. doi:10.1136/jitc-2020-001800

Oh, S. H., An, D. B., Kim, T. H., and Lee, J. H. (2016). Wide-range Stiffness Gradient PVA/HA Hydrogel to Investigate Stem Cell Differentiation Behavior. Acta Biomater. 35, 23-31. doi:10.1016/j.actbio.2016.02.016

Oliva, N., Conde, J., Wang, K., and Artzi, N. (2017). Designing Hydrogels for OnDemand Therapy. Acc. Chem. Res. 50 (4), 669-679. doi:10.1021/ acs.accounts.6b00536

Overstreet, D. J., Dutta, D., Stabenfeldt, S. E., and Vernon, B. L. (2012). Injectable Hydrogels. J. Polym. Sci. B Polym. Phys. 50 (13), 881-903. doi:10.1002/polb.23081

Pal, K., Singh, V. K., Anis, A., Thakur, G., and Bhattacharya, M. K. (2013). Hydrogel-Based Controlled Release Formulations: Designing Considerations, Characterization Techniques and Applications. Polymer-Plastics Technology Eng. 52 (14), 1391-1422. doi:10.1080/03602559.2013.823996

Palucka, K., and Banchereau, J. (2012). Cancer Immunotherapy via Dendritic Cells. Nat. Rev. Cancer 12 (4), 265-277. doi:10.1038/nrc3258

Qiu, H., Guo, H., Li, D., Hou, Y., Kuang, T., and Ding, J. (2020). Intravesical Hydrogels as Drug Reservoirs. Trends Biotechnol. 38 (6), 579-583. doi:10.1016/ j.tibtech.2019.12.012

Rastogi, P., and Kandasubramanian, B. (2019). Review of Alginate-Based Hydrogel Bioprinting for Application in Tissue Engineering. Biofabrication 11 (4), 042001. doi:10.1088/1758-5090/ab331e

Rizzo, F., and Kehr, N. S. (2021). Recent Advances in Injectable Hydrogels for Controlled and Local Drug Delivery. Adv. Healthc. Mater. 10 (1), 2001341. doi:10.1002/adhm.202001341

Salah, M., Tayebi, L., Moharamzadeh, K., and Naini, F. B. (2020). Three-dimensional Bio-Printing and Bone Tissue Engineering: Technical Innovations and Potential Applications in Maxillofacial Reconstructive Surgery. Maxillofac. Plast. Reconstr. Surg. 42 (1), 18. doi:10.1186/s40902-020-00263-6

Seliktar, D. (2012). Designing Cell-Compatible Hydrogels for Biomedical Applications. Science 336 (6085), 1124-1128. doi:10.1126/science.1214804

Shimizu, T., Kishida, T., Hasegawa, U., Ueda, Y., Imanishi, J., Yamagishi, H., et al. (2008). Nanogel DDS Enables Sustained Release of IL-12 For Tumor Immunotherapy. Biochem. Bioph. Res. Co. 367, 330-335. doi:10.1016/ j.bbrc.2007.12.112
Simona, B. R., Hirt, L., Demkó, L., Zambelli, T., Vörös, J., Ehrbar, M., et al. (2015). Density Gradients at Hydrogel Interfaces for Enhanced Cell Penetration. Biomater. Sci. 3 (4), 586-591. doi:10.1039/c4bm00416g

Song, H., Huang, P., Niu, J., Shi, G., Zhang, C., Kong, D., et al. (2018). Injectable Polypeptide Hydrogel for Dual-Delivery of Antigen and TLR3 Agonist to Modulate Dendritic Cells In Vivo and Enhance Potent Cytotoxic T-Lymphocyte Response against Melanoma. Biomaterials 159, 119-129. doi:10.1016/j.biomaterials.2018.01.004

Tan, H., and Marra, K. G. (2010). Injectable, Biodegradable Hydrogels for Tissue Engineering Applications. Materials 3 (3), 1746-1767. doi:10.3390/ma3031746

Tang, T., Huang, X., Zhang, G., Hong, Z., Bai, X., and Liang, T. (2021). Advantages of Targeting the Tumor Immune Microenvironment over Blocking Immune Checkpoint in Cancer Immunotherapy. Sig Transduct Target. Ther. 6 (1), 72. doi:10.1038/s41392-020-00449-4

Tie, S., Zhang, X., Wang, H., Song, Y., and Tan, M. (2020). Procyanidins-Loaded Complex Coacervates for Improved Stability by Self-Crosslinking and Calcium Ions Chelation. J. Agric. Food Chem. 68 (10), 3163-3170. doi:10.1021/acs.jafc.0c00242

Tu, Y., Chen, N., Li, C., Liu, H., Zhu, R., Chen, S., et al. (2019). Advances in Injectable Self-Healing Biomedical Hydrogels. Acta Biomater. 90, 1-20. doi:10.1016/j.actbio.2019.03.057

Vigata, M., Meinert, C., Hutmacher, D. W., and Bock, N. (2020). Hydrogels as Drug Delivery Systems: A Review of Current Characterization and Evaluation Techniques. Pharmaceutics 12 (12), 1188. doi:10.3390/pharmaceutics 12121188

Vojtova, L., Michlovska, L., Valova, K., Zboncak, M., Trunec, M., Castkova, K., et al. (2019). The Effect of the Thermosensitive Biodegradable PLGA-PEGPLGA Copolymer on the Rheological, Structural and Mechanical Properties of Thixotropic Self-Hardening Tricalcium Phosphate Cement. Ijms 20 (2), 391. doi:10.3390/ijms20020391

Weiden, J., Tel, J., and Figdor, C. G. (2018). Synthetic Immune Niches for Cancer Immunotherapy. Nat. Rev. Immunol. 18 (3), 212-219. doi:10.1038/nri.2017.89

Wu, Y., Li, Q., Shim, G., and Oh, Y.-K. (2021). Melanin-loaded CpG DNA Hydrogel for Modulation of Tumor Immune Microenvironment. J. Controlled Release 330, 540-553. doi:10.1016/j.jconrel.2020.12.040

Xie, Z., Shen, J., Sun, H., Li, J., and Wang, X. (2021). Polymer-based Hydrogels with Local Drug Release for Cancer Immunotherapy. Biomed. Pharmacother. 137, 111333. doi:10.1016/j.biopha.2021.111333

Yang, F., Shi, K., Jia, Y.-p., Hao, Y., Peng, J.-r., and Qian, Z.-y. (2020). Advanced Biomaterials for Cancer Immunotherapy. Acta Pharmacol. Sin 41 (7), 911-927. doi:10.1038/s41401-020-0372-z

Yang, P., Song, H., Qin, Y., Huang, P., Zhang, C., Kong, D., et al. (2018). Engineering Dendritic-Cell-Based Vaccines and PD-1 Blockade in SelfAssembled Peptide Nanofibrous Hydrogel to Amplify Antitumor T-Cell Immunity. Nano Lett. 18 (7), 4377-4385. doi:10.1021/acs.nanolett.8b01406

Yu, S., Wang, C., Yu, J., Wang, J., Lu, Y., Zhang, Y., et al. (2018). Injectable Bioresponsive Gel Depot for Enhanced Immune Checkpoint Blockade. Adv. Mater. 30 (28), 1801527. doi:10.1002/adma.201801527

Zeng, Y., Xiang, Y., Sheng, R., Tomás, H., Rodrigues, J., Gu, Z., et al. (2021). Polysaccharide-based Nanomedicines for Cancer Immunotherapy: A Review. Bioactive Mater. 6 (10), 3358-3382. doi:10.1016/j.bioactmat.2021.03.008

Zhang, R., Billingsley, M. M., and Mitchell, M. J. (2018). Biomaterials for VaccineBased Cancer Immunotherapy. J. Controlled Release 292, 256-276. doi:10.1016/ j.jconrel.2018.10.008

Zou, W. (2006). Regulatory T Cells, Tumour Immunity and Immunotherapy. Nat. Rev. Immunol. 6 (4), 295-307. doi:10.1038/nri1806

Conflict of Interest: The authors declare that the research was conducted in the absence of any commercial or financial relationships that could be construed as a potential conflict of interest.

The handling editor declared a shared affiliation, though no other collaboration, with several of the authors RC, QW, YX and DL at the time of the review.

Copyright (c) $2021 \mathrm{Cui}, \mathrm{Wu}$, Wang, Zheng, $\mathrm{Ou}, \mathrm{Xu}, \mathrm{Qu}$ and Li. This is an open-access article distributed under the terms of the Creative Commons Attribution License (CC BY). The use, distribution or reproduction in other forums is permitted, provided the original author(s) and the copyright owner(s) are credited and that the original publication in this journal is cited, in accordance with accepted academic practice. No use, distribution or reproduction is permitted which does not comply with these terms. 